\title{
Intelligente Lösungen im klinischen Alltag, die den Informationsfluss verbessern: Checkpad MED auf dem iPad
}

\author{
Rüdiger Lohmann, Almut Tempka, Norbert Südkamp
}

\section{Zusammenfassung}

Der Austausch und eine hohe Verfügbarkeit wichtiger Informationen und Daten innerhalb klinischer Prozesse sind entscheidend für komplikationsfreie Abläufe im Krankenhausmanagement und insbesondere bei der Entscheidungssicherheit in der Behandlung von Patienten. Trotz hochentwickelter papierloser Informations- und Kommunikationstechnologien, die den Klinikalltag erleichtern können, werden Informationen oder Aufgaben von Ärzten und Pflegekräften überwiegend noch handschriftlich dokumentiert. Diagnostische Informationen sind in unterschiedlichen Softwaresystemen und meistens nur an stationären Arbeitsplätzen abzurufen. Dies führt häufig zu Informationsverlusten und einer erhöhten Fehleranfälligkeit der Prozessschritte im medizinischen Behandlungsablauf. Im schlimmsten Fall kann das den Patienten gefährden, aber auch Krankenhauskosten in die Höhe treiben. Eine Möglichkeit, den Informationsfluss zwischen den Aktionären im Krankenhaus zu verbessern, bildet die Software Checkpad MED auf dem iPad, die als eine digitale Patientenakte fungiert und in der Lage ist, Workflows, Aufgaben und Kommunikation der Nutzer zu managen. Wie der Informationsfluss im Großteil der deutschen Kliniken heute erfolgt und wie dieser zukünftig durch Mobile Devices, wie Checkpad MED auf dem iPad, digital unterstützt und verbessert werden könnte, soll im folgenden Artikel beispielhaft dargestellt werden.

\section{Intelligent Solutions to Improve the Flow of Information in Clinical Practice: Checkpad MED for iPad}

The exchange and high availability of important information and data from clinical procedures are essential for error-free processes in hospital management and, in particular, the sureness of decisions concerning the treatment of patients. In spite of sophisticated paperless information and communication technologies that can facilitate the daily clinical practice, information or tasks of doctors and nurses are mostly documented in manuscript form. Also diagnostic information often has to be retrieved in different software systems and can only be obtained at fixed workstations. This leads to a loss of information and an increased susceptibility to errors in the procedural steps in the medical treatment process. In the worst case, this can endanger the patient, but it also drives up hospital costs. One way to improve the flow of information between shareholders in the hospital constitutes the Software Checkpad MED for iPad, which functions as an electronic health record (EHR). It is able to manage workflows, tasks and communication of the user. How the flow of information in the majority of German hospitals is done today and how this could be digitally supported and improved by mobile devices like Checkpad MED for iPad in the future, are exemplified in the present article.

\section{Einleitung \\ IT im Krankenhaus}

Die Informationstechnologie hat im ärztlichen Alltag im Rahmen der Einführung von Fallpauschalen und Sonderentgelten Einzug gehalten. Ab diesem Zeitpunkt war es notwendig, zu jedem Patienten sowohl Diagnosen als auch Prozeduren in kodierter Form zu dokumentieren und an die Kostenträger zu übermitteln. Dementsprechend wurden die IT-Systeme entwickelt. Die typische Funktionalität bestand darin, dass eine Liste von Fällen einer Leistungsstelle (z.B. Station) angezeigt wurde. $\mathrm{Zu}$ jedem Fall konnten dann Diagnosen und Prozeduren hinzugefügt werden. Zur Verschlüsselung wurde zunächst v.a. das Programm DIACOS ${ }^{\circledR}$ von der Firma ID genutzt, später folgte das Programm KODIP ${ }^{\circledR}$ von SBG. KODIP ${ }^{\circledR}$ wurde im Jahr 2006 von dem Unternehmen 3M übernommen. Fortschrittliche Software zeigte zu diesem Zeitpunkt schon Fälle ohne eine kodierte Diagnose an, was die ersten Schritte zu einer Workflowsoftware im Bereich der ärztlichen Dokumentation darstellte. Diagnosen und Prozeduren wurden seither an die Verwaltung übermittelt, die aus den Daten eine Rechnung erstellte und diese schließlich an die Kostenträger versendete.

Neben der Dokumentation wurden ITSysteme vermehrt als Auskunftssysteme genutzt. Gerade Laborwerte und Bildgebung stehen heute fast flächendeckend auf IT-Systemen zur Verfügung. Der nächste Schritt der IT-Entwicklung in Krankenhäusern war die Anforderung von Leistungen über Bildschirmmasken. Nicht immer hat sich durch das Ausfüllen von Feldern am PC die Geschwindigkeit von Prozessen verbessert. 


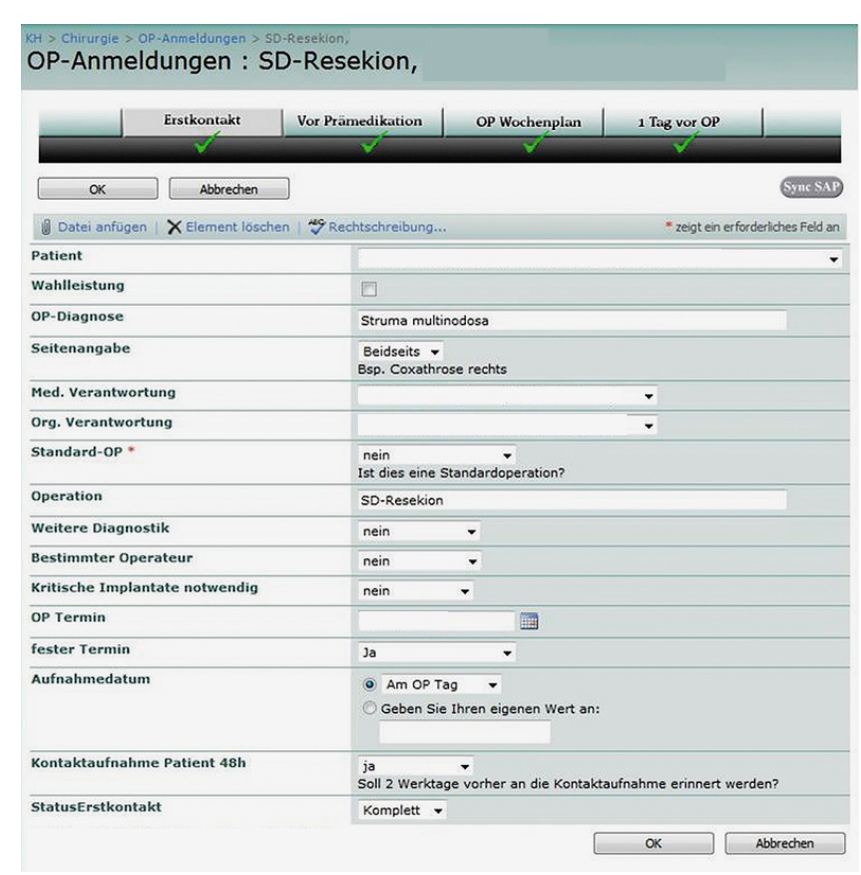

Abb. 1 Datenerfassung bei Erstkontakt Patient.*

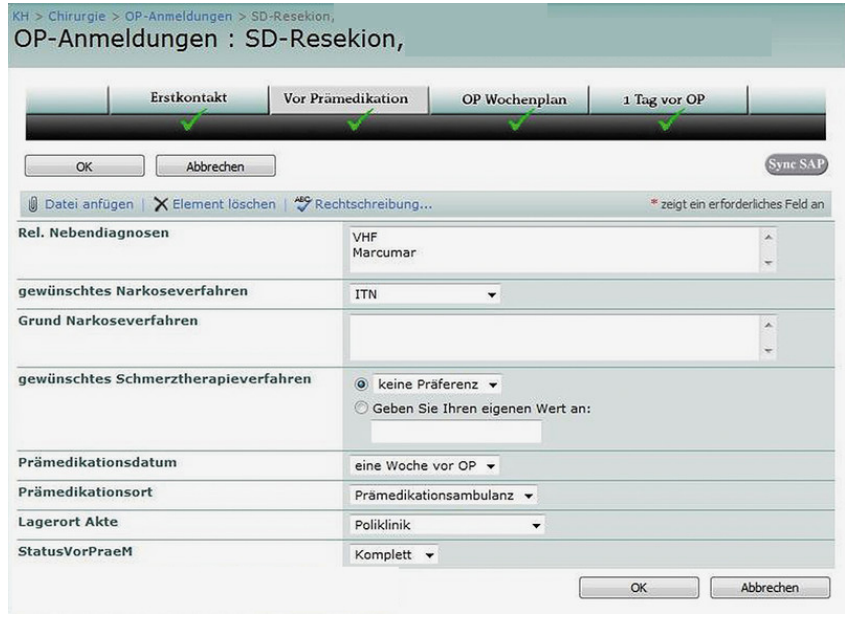

Abb. 2 Datenerfassung vor Prämedikation.

\footnotetext{
* Die in den Abbildungen verwendeten Namen und Daten sind lediglich fiktiv.
}

Heute lassen sich die IT-Systeme in die folgenden typischen Bereiche einteilen:

- Dokumentationssysteme (besonders Diagnosen- und Prozedurendokumentation)

- Auskunftsysteme (Labor, Radiologie, Befunde)

- Anforderungssysteme

Durch die primäre Notwendigkeit, bei der Einführung von IT im Krankenhaus besonders Informationen für die Abrechnung zu erfassen, hat der Bereich, der die Mitarbeiter in der Medizin am meisten beschäftigt, oft eine etwas stiefmütterliche Rolle gespielt. Der Blick in die Kitteltasche eines Arztes oder einer Pflegekraft zeigt, dass die digitale Unterstützung des Arbeitsalltags noch viel Spielraum nach oben zulässt. So findet sich in fast jeder Tasche mindestens ein Zettel, auf dem Listen von Patienten, wichtige Informationen oder Aufgaben handschriftlich dokumentiert sind. Diese Listen werden oft täglich neu erstellt oder über einige Tage weitergeführt. Gerade bei den mittlerweile sehr kurzen Liegedauern im Krankenhaus führt dies zu viel durchgestrichenem Text $[1,2]$.

Die Herausforderung der IT ist nun, auch Arbeitsprozesse in der Medizin zu unterstützen [1]. Eine der größten Schwierigkeiten besteht jedoch darin, dass die Erbringung von medizinischen Leistungen im Krankenhaus fast immer mit einem sehr hohen mobilen Anteil erfolgt. Ärzte bewegen sich zwischen Besprechungsraum, Röntgendemoraum, Ambulanz,
Funktion, OP, Normalstation und Intensivstation praktisch pausenlos hin und her. Das Pflegepersonal hat als typischen Aktionsradius: Stützpunkt, Arztzimmer, Verbandszimmer, Funktion, OP, andere Stationen und die Patientenzimmer. Nur ein Teil der Zeit wird am Schreibtisch, auf dem oft der PC steht, verbracht. Da die Anzahl der PCs auf einer Station nicht nur durch die Kosten der Hardware und der Netze, sondern auch durch den vorhandenen Platz begrenzt ist, ist das Warten auf einen freien Zeitslot am PC heute oft klinische Realität. Viele Informationen stehen in der Zeit, in der ein Mitarbeiter nicht auf einen Bildschirm schauen kann, nicht oder nur als Erinnerung zur Verfügung.

\section{Prozesse im Krankenhaus}

In der Beschreibung der Tätigkeit eines Arztes auf einer operativen Station lassen sich typische Prozesse im Ablauf eines Tages definieren. Diese Prozesse sind in vielen Krankenhäusern sehr ähnlich. Einige typische Prozesse sind hier stichpunktartig aufgeführt:

\section{Morgens auf der Station}

\section{Information}

Informationen über Veränderungen während der Nacht:

- Welche Patienten wurden neu aufgenommen?

- Wer wurde verlegt?

- Wer hatte nicht zu erwartende Komplikationen (z.B. Nachblutung mit
Nach-OP, Temperatur, Schmerzen) mit konsekutiver Konsequenz in Form von Diagnostik oder Therapie?

- Von wem und wie, mit welchem Ergebnis gemanagt?

\section{Entscheidung}

- Muss am geplanten Tagesablauf etwas geändert werden?

- ggf. Durchführung der Änderung

\section{Röntgenbesprechung}

\section{Information}

- Kurzbericht zum Patienten (Anamnese, Diagnostik, Therapie)

- Vorstellung der Bildgebung

\section{Entscheidung}

- weitere Diagnostik oder Therapie

Visite auf der Station

\section{Information}

- Sichtung der neuen Informationen seit letzter Übergabe/Visite

- Bericht Pflege

- Befragung Patient

- Untersuchung Patient

- Informationsweitergabe an Patienten

\section{Entscheidung}

- weitere Diagnostik oder Therapie

- Entlassungsplanung

Diese typischen Prozesse sind durch Informationsanalyse, Informationserfassung mit Dokumentation, Entscheidungsfindung und Entscheidungsdoku- 


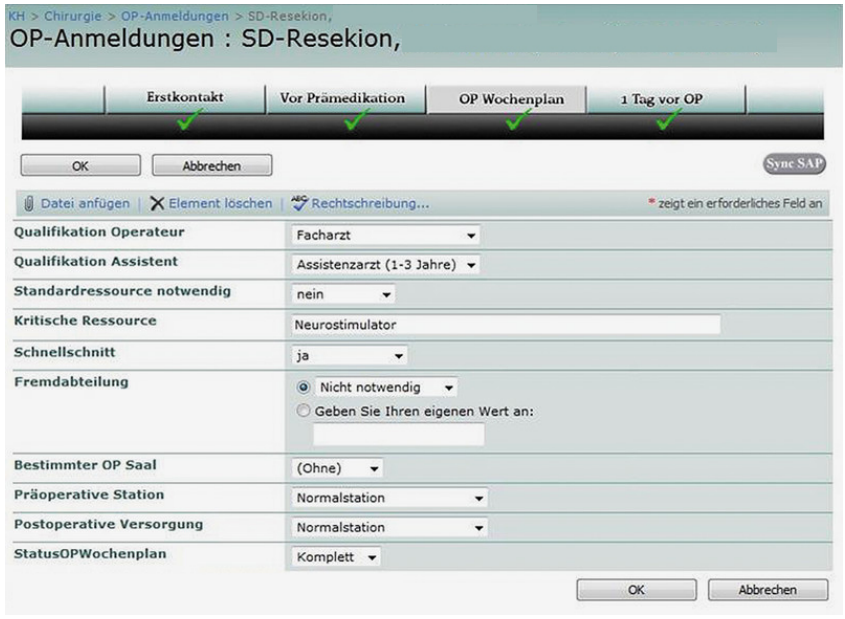

Abb. 3 Datenerfassung vor Erstellung des OP-Wochenplans.

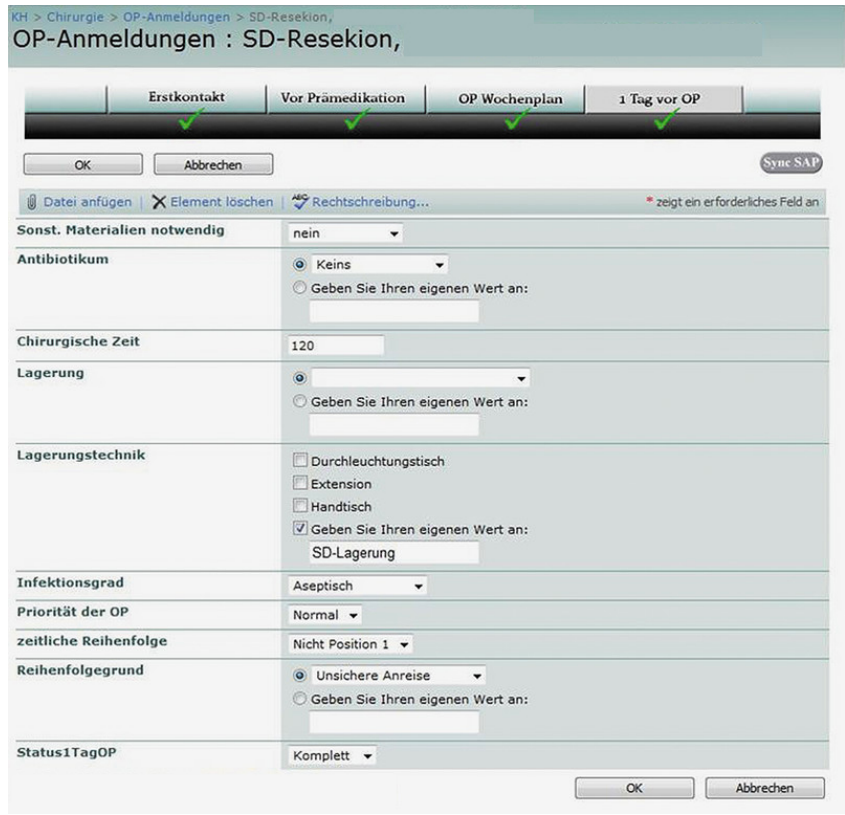

Abb. 4 Datenerfassung am Tag vor der OP. mentation gekennzeichnet. Die nicht lückenlose Dokumentation dieser Schritte kann dann zu Prozessbrüchen führen, die das Krankenhaus primär Geld kosten, den Patienten im schlimmsten Fall jedoch auch gefährden können.

\section{Klinische Beispiele zum Informationsfluss}

\section{Chirurgie: OP von der Anmeldung bis zum Hautschnitt}

Im Rahmen eines Projekts in der Klinik für Unfall- und Wiederherstellungschirurgie der Charité, Campus Virchow-Klinikum (Prof. Norbert Haas) wurde der gesamte Prozess der OP-Anmeldung dokumentiert. Ziel war eine Informationserfassung und -weitergabe, die eine für den Patienten möglichst erfolgreiche und risikoarme Operation, bei möglichst schonendem Ressourceneinsatz, ermöglicht.

Soll ein Patient operiert werden, so sind zunächst Informationen zu sammeln, um die Indikation $\mathrm{zu}$ bestätigen. Im nächsten Schritt wird nach Kontraindikationen zum geplanten Vorgehen gesucht. So werden Patienten vor der Operation der Anästhesie vorgestellt, um die Narkosefähigkeit prüfen zu lassen. Diese Schritte lassen sich in präoperative Aufgaben, Aufgaben am Tag vor der OP und Aufgaben am Tag der OP gliedern. Das typische Vorgehen bei elektiven Patienten wird nachfolgend beschrieben.

\section{Prozedere präoperativ}

Vorstellung des Patienten, Erstkontakt Alle elektiven Patienten werden in der Poliklinik der Abteilung gesehen. Die Poliklinik/Ambulanz wird tageweise von Ärzten der verschiedenen Teams der Abteilung besetzt.

\section{Diagnostik}

Erforderliche diagnostische Maßnahmen werden, so weit wie möglich, im ambulanten Vorfeld veranlasst.

\section{OP-Indikation}

Die OP-Indikation wird vom Poliklinikarzt ggf. nach Rücksprache mit einem Oberarzt gestellt. Kontraindikationen müssen ebenfalls in diesem Zusammenhang ausgeschlossen sowie Behandlungsalternativen und deren Vor- und Nachteile dargelegt werden.

\section{OP- und Therapieplanung}

Jedes Team führt einen digitalen Terminkalender im Krankenhausinformationssystem. Hier sind neben den Patientendaten die Diagnose, die geplante Operation und in Einzelfällen besondere Materialien, Implantate, spezielle Lagerungen und Ähnliches vermerkt.

\section{Chirurgische Aufklärung}

Die chirurgische Aufklärung elektiver Patienten erfolgt ambulant.

\section{Prämedikation}

Die Prämedikation elektiver Patienten erfolgt in der Prämedikationsambulanz.
Nur in schweren oder wider Erwarten akuten Fällen werden die Patienten einen Tag vor der geplanten Operation aufgenommen und die Prämedikation dann auf der Station durchgeführt.

\section{Kostenübernahme klären}

Die korrekte Kostenübernahme muss bei der stationären Aufnahme kontrolliert werden.

\section{Prozedere am Tag vor OP}

OP-Anmeldung und OP-Plan-Erstellung Der Stationsarzt führt am Morgen des Tages vor der geplanten OP eine OP-Anmeldung durch. Dabei werden die elektiven Eingriffe aus dem digitalen Terminkalender übernommen sowie nicht geplante Eingriffe mit dringlicher OP-Indikation aus der Stationsliste berücksichtigt.

\section{Typische OP-Komplikationen vor dem Hautschnitt}

Bei der Analyse der Gründe für einen Ausfall einer OP, Verschiebung oder Verzögerung im OP-Ablauf, wurde eine Reihe von Gründen identifiziert. Im Zeitraum von der Vorstellung des Patienten bis zur endgültigen OP-Anmeldung waren dies oft nicht dokumentierte Zusatzinformationen (Nebendiagnosen, präferiertes Narkoseverfahren oder sehr selten ein bestimmtes notwendiges Implantat). Im Rahmen der OP-Anmeldung wurden als Probleme nicht angemeldete notwendige Ressourcen (bestimmter Tisch, Turm oder Saal), fehlende Infor- 


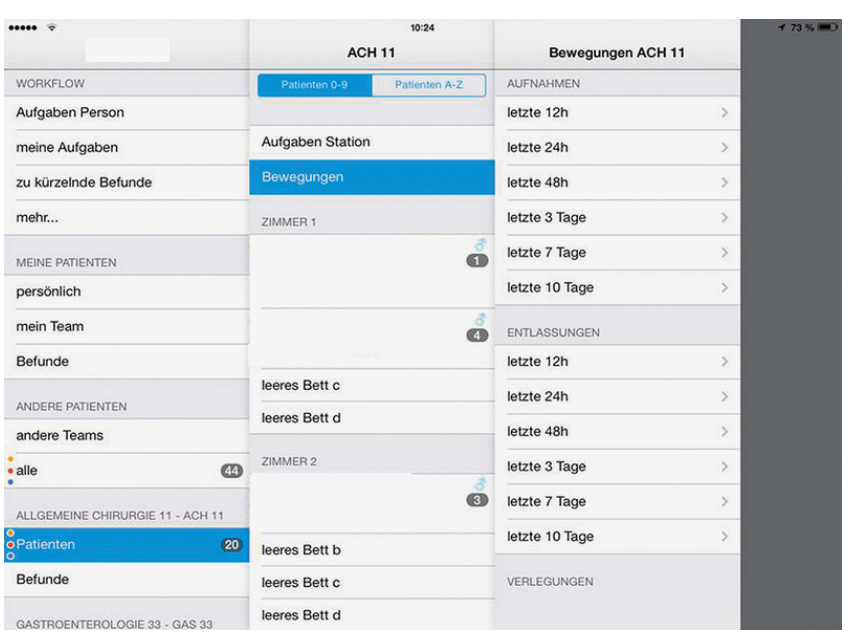

Abb. 5 Anzeige Patientenbewegungen einer Leistungsstelle in Checkpad MED auf dem iPad.

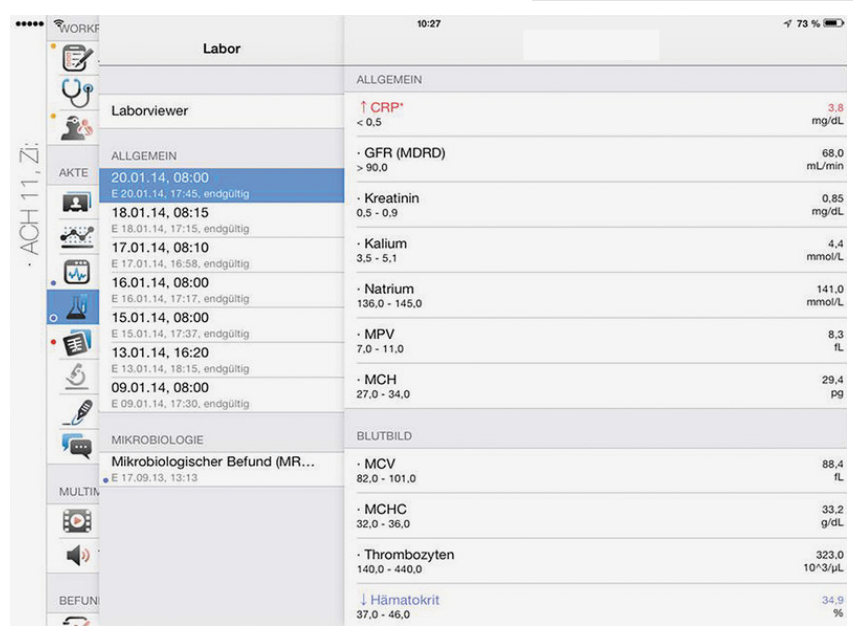

Abb. 6 Anzeige Laborwerte eines Befunds eines Patienten. mationen über den Zustand des Patienten (Infektionsgrad oder Allergien), Beteiligung anderer Abteilungen oder fehlende Informationen über die Station des Patienten, auf die er nach der Operation verlegt werden soll, als Ursachen für eine Verzögerung im Ablauf identifiziert. Mittels dieser Informationen wurde ein Soll-Datenbestand zusammengestellt, dessen Vorhandensein bei sorgfältiger Informationserfassung die meisten Ablaufverzögerungen vermeiden sollte. Für die meisten Kliniken wird nur ein Teil dieser möglichen Datenfelder relevant sein. Folgend werden jedoch die möglichen Datenfelder kurz beschrieben.

\section{Informationsbedarf bis zur OP}

Bei Erstkontakt Patient (Abb. 1)

- Stammdaten Patient (Name, Vorname, Geburtsdatum, Geschlecht, Telefonnummern [mobil und Festnetz])

- Wahlleistung ja/nein

- OP-Diagnose

- Seitenangabe zum operativen Eingriff

- medizinische Verantwortung für die OP (z.B. OA)

- organisatorische Verantwortung für die OP (z. B. Assistenzarzt)

- Standard-OP (Auswahl füllt weitere Felder automatisch aus)

- weitere Diagnostik

- bestimmter Operateur, falls ja: Angabe des Operateurs

- vorab zu bestellende Implantate

- geplanter OP-Termin

- OP-Termin fest oder vonseiten des Patienten verschiebbar

- geplantes Aufnahmedatum in Relation zur OP (OP-Tag, Tag vor OP)

- Kontaktaufnahme mit Patient z.B. 48 Stunden vor der OP geplant
Vor Prämedikation (Abb. 2)

- relevante Nebendiagnosen

- gewünschtes Narkoseverfahren mit Grund

- gewünschtes Schmerztherapieverfahren mit Grund

- geplantes Prämedikationsdatum

- Prämedikationsort

- Lagerort der Akte

Bei Erstellung des OP-Wochenplans (Abb. 3)

- Qualifikation Operateur, wenn nicht bestimmter Operateur

- Qualifikation Assistenz

- Eingabe Standardressourcen (Bildwandler, Endoskopieturm etc.)

- Schnellschnitt ja/nein

- notwendige Fremdabteilungen inkl. gewünschter Leistung

- bestimmter OP-Saal

- präoperative Station oder Ambulanz

- postoperative Station oder Ambulanz

Am Tag vor der OP (Abb. 4)

- besondere Materialwünsche

(z.B. Schiene zur Nachversorgung)

- Antibiotikagabe

- OP-Zeit

- Lagerungsart

- notwendige Lagerungstechnik

- Infektionsgrad

- Priorität im OP

- gewünschte zeitliche Reihenfolge (z.B. an erster Stelle bei Latexallergie)

\section{OP-Vorbereitung mobil}

Diese Art der Informationssammlung auf einem stationären PC kann den Prozess der OP-Anmeldung deutlich transparenter machen und eine Vielzahl von Verzögerungen im Ablauf verhindern. Dies kann jedoch nur als Zwischenschritt gesehen werden.

Eine gute OP-Vorbereitung bedingt, dass auch eine Kommunikation zwischen unterschiedlichen Bereichen und z. B. dem organisierenden Assistenzarzt und dem verantwortlichen Oberarzt schnell möglich ist.

Liegen die Daten nur in Form von Masken im PC vor, so kann die Kommunikation nur zu den Zeitpunkten stattfinden, an denen jemand vor einem Rechner sitzt. In der täglichen Arbeit ist dies jedoch meist nicht der Fall. Auf der Station ergibt sich zusätzlich das Problem, dass die vorhandenen Rechner von den verschiedenen Fachdisziplinen oft geteilt werden müssen. So benötigen z.B. Stationsärzte, Pflegepersonal, Sozialdienstmitarbeiter, Konsiliarien, Physiotherapeuten etc. Zugang zu Patientendaten.

Die Lösung dieses Dilemmas kann in mobilen Devices liegen, die es ermöglichen, dass die jeweiligen Daten immer und überall zur Verfügung stehen und eine Kommunikation jederzeit möglich ist. Heute sind Programme wie Facebook, Twitter, Instagram, WhatsApp, iMessage oder Tumblr aus dem täglichen Leben, gerade junger Menschen, nicht mehr wegzudenken. Diese Programme erlauben es, asynchron zu kommunizieren. Dem Gegenüber wird die Möglichkeit gegeben, zu einem ihm angenehmen Zeitpunkt, auf Fragen zu antworten. Dahingegen wird im Krankenhaus versucht, möglichst viel synchron zu kommunizieren. Ein typisches Beispiel sind Telefonanrufe, die das Gegenüber aus seiner aktuellen Arbeit herausreißen und es zwingen, sich einem anderen 


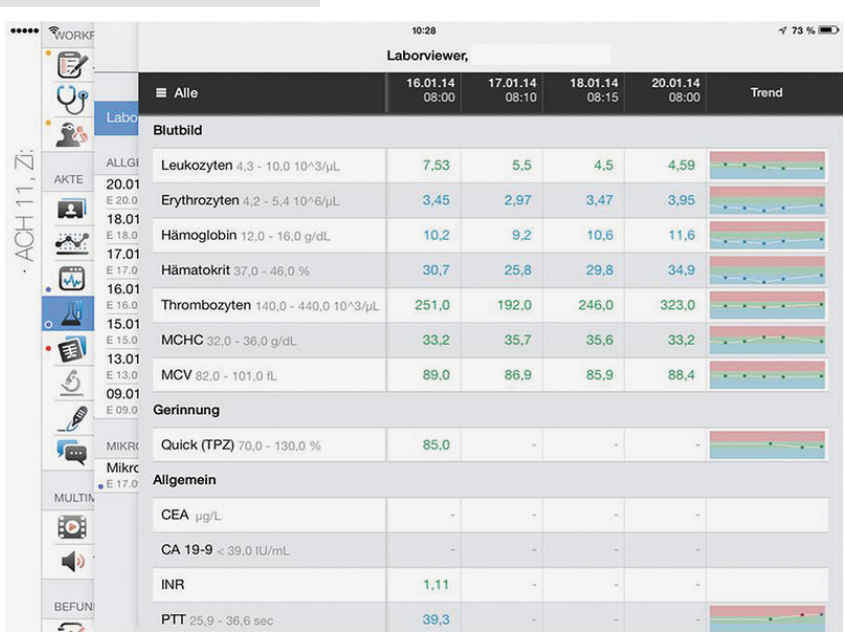

Abb. 7 Anzeige Kumulativbefunde Labor Patienten.

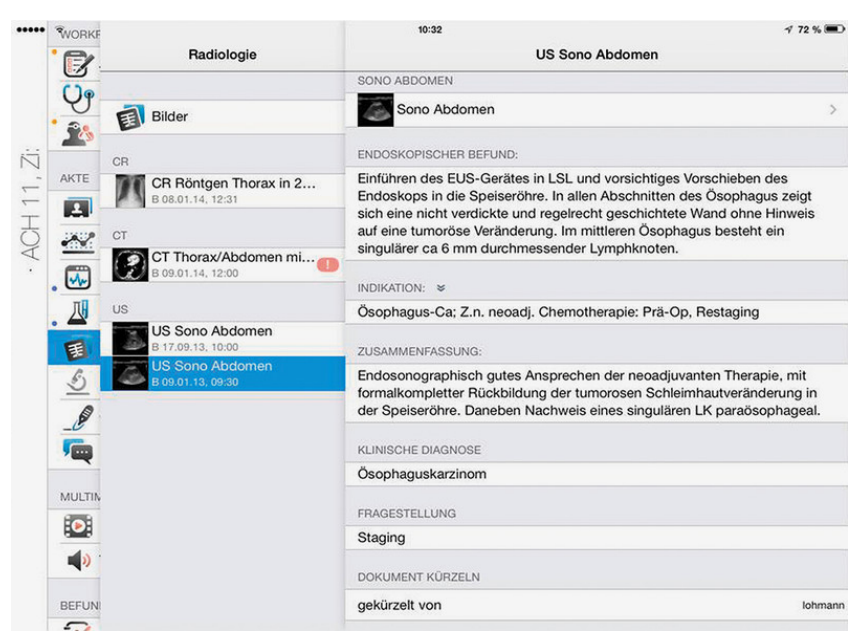

Abb. 8 Markieren von gesehenen Befunden.
Thema sofort zuzuwenden. In den meisten Fällen ist jedoch eine sofortige Antwort gar nicht nötig.

Aus diesem Grund wurde Checkpad MED entwickelt, eine Software, welche die Vorteile der modernen Kommunikation mit den Bedürfnissen in der Medizin kombiniert.

Hauptaufgabe ist zum einen, die Informationen einer Krankenakte an jedem Ort, zu jeder Zeit, auch für mehrere an der Behandlung beteiligte Personen, verfügbar zu machen, zum anderen soll aber auch die Kommunikation asynchron ermöglicht werden. So sollen Aufgaben an jemanden gestellt werden können, die sich diese Person z.B. zwischen zwei OPs ansehen und ggf. abarbeiten kann. Diese Software ist z. B. am Universitätsklinikum Freiburg in der Klinik für Orthopädie und Unfallchirurgie (Prof. Norbert Südkamp) seit einiger Zeit im Einsatz (Abb. 5).

Zum Verständnis soll hier der Zugriff auf die Krankenakte am Beispiel von Laborwerten und Röntgenbildern demonstriert werden. Anschließend wird das Verfahren zum Stellen und Abarbeiten von Aufgaben sowie zur Anzeige von Patientenübersichten vorgestellt.

Das System liest im ersten Schritt die Stammdaten eines Krankenhausfalls ein und stellt aus der Organisationsstruktur eines Krankenhauses (Abteilungen, Stationen, Zuordnung der Personen zu Leistungsstellen) einen Soll-Datenbestand von Patienten für einen einzelnen Nutzer zusammen. Diese Daten werden auf ein iOS-Device wie z.B. ein iPad gespiegelt. Damit kann zunächst die komplette
Stationsbelegung, ggf. mit Zuordnung der Zimmer und der Betten im Zimmer, angezeigt werden. Bei Veränderungen der Belegung werden diese bei Kontakt z.B. zum WLAN sofort auf dem iPad nachvollzogen. Sollte gerade kein Kontakt bestehen, so wird die Änderung in eine Warteschlange gestellt und beim nächsten Kontakt des Geräts zum Netz automatisch durchgeführt. Diese Informationen können so aufbereitet werden, dass auch Listen mit Aufnahmen, Verlegungen und Entlassungen, in definierten Zeiträumen, angezeigt werden können.

Als nächstes werden Laborwerte aus dem Laborsystem übernommen und so aufbereitet, dass sie auf dem iPad dargestellt werden können. Vorteilhaft dabei ist, dass auch schon Laboranforderungen kommuniziert werden können, sodass eine Laboruntersuchung, von der Anforderung über den Materialeingang im Labor und die vorläufigen Werte bis zum endgültigen Befund, fast ohne Zeitverzögerung auf dem iPad zur Verfügung gestellt werden kann. Dabei ist es möglich, sowohl Einzelbefunde als auch Kumulativbefunde mit grafischer Darstellung zur Anzeige zu bringen (Abb. 6 und 7).

Im nächsten Schritt werden Befunde aus den Krankenhausinformationssystemen übernommen und in einer frei konfigurierbaren Krankenakte nach Kategorien und absteigend nach Erstellungsdatum angezeigt. Einzelne Befunde können als wichtig markiert werden, sodass jemand, der am Vortag z. B. wegen Nachtdienst nicht anwesend war, sofort Zugriff auf relevante Informationen hat. Bei den Befunden gibt es weiterhin die Möglich- keit, diese als gelesen zu markieren (Abb. 8). Diese Information wird wiederum zurück in die Datenbank gespielt und auf die anderen iPads gespiegelt.

Etwas komplexer ist die Übernahme von Röntgenbildern und Schnittbilddiagnostik. Diese Informationen liegen meist im DICOM-Format in einem sog. PACS (Picture Archiving and Communication System) vor. Für das Auffinden von Bildern gibt es zwei Möglichkeiten. Die erste Möglichkeit besteht darin, in regelmäßigen Abständen nach neuen Bildern von aktuell einliegenden Patienten über die Fallnummer zu suchen. Als Suchergebnis erhält man eine Liste von Studien. Jede Studie kann dann einzeln geladen werden. Dieses Verfahren setzt das System abhängig von der Häufigkeit der Suchanfragen und der Anzahl der Fallnummern unter Last, sodass es gut ausbalanciert werden muss. Der elegantere Weg ist, dass bei jeder Untersuchungsanforderung bereits ein Leerbefund dieser Untersuchung erstellt und z.B. per HL7 kommuniziert wird. Hat die Untersuchung nun stattgefunden, so wird eine neue Version des Untersuchungsbefunds erstellt, die zunächst additiv die Studienidentifikationsnummer enthält. Damit kann nach den Bildern dieser Untersuchung gezielt gesucht und Untersuchungsbefunde und dazugehörige Bilder können auf dem iPad zusammen angezeigt werden. Sobald der Befund dokumentiert wurde, kann eine neue Version des vorher leeren Dokuments erzeugt und übertragen werden.

Die geladenen Bilder sollten für die Anzeige auf dem iPad konfiguriert werden. So kann ein iPad nur 8 Bit in der Oberfläche anzeigen, DICOM-Bilder sind aber 


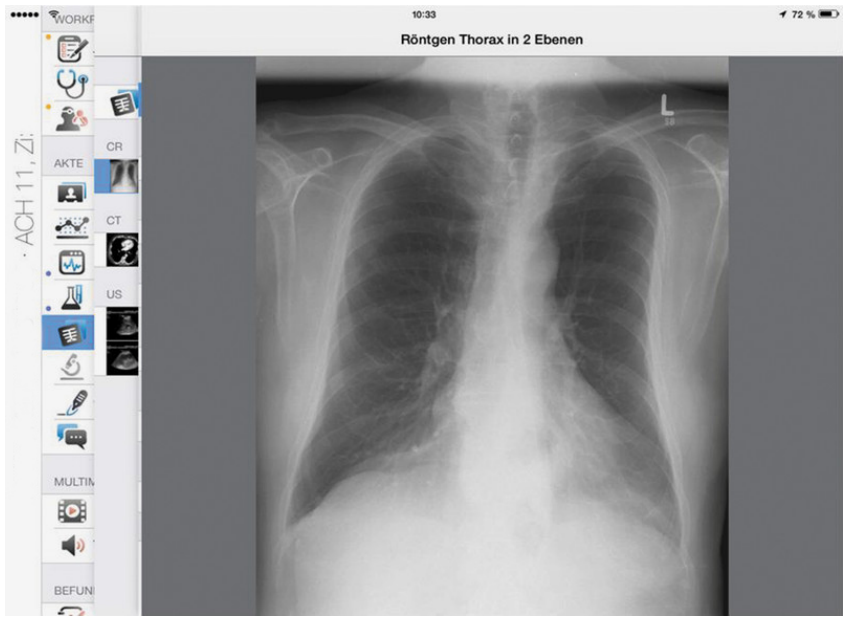

Abb. 9 Anzeige Röntgenthorax auf dem iPad.

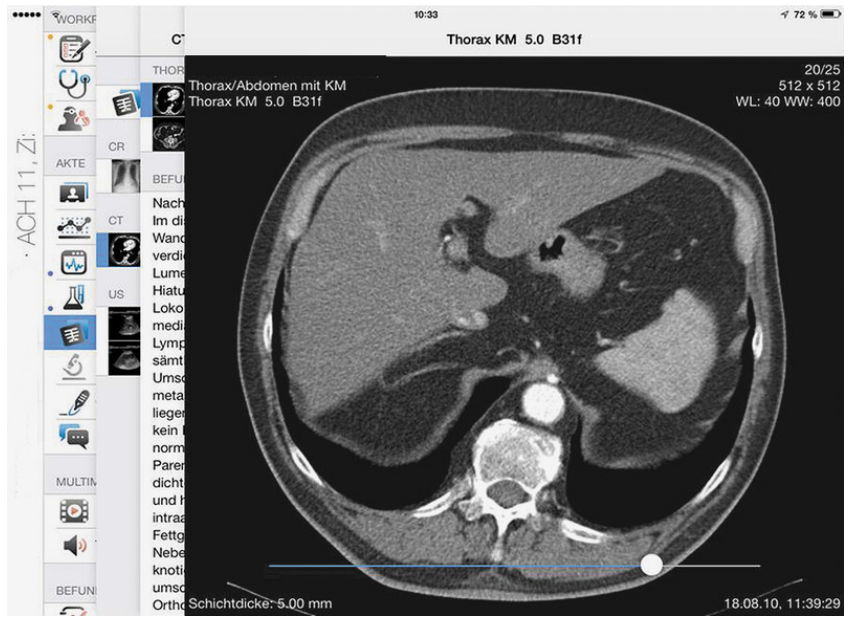

Abb. 10 Anzeige CT Abdomen auf dem iPad. meist in 16 Bit kodiert. Auch Farbraum und Auflösung sollten den Möglichkeiten des mobilen Geräts angepasst werden. Das gleiche Vorgehen empfehlen wir bei CTs und MRTs. Es bietet sich auch an, nicht jedes einzelne Bild, sondern z.B. jedes zweite Bild zu verwenden, um die Menge an Daten, die auf ein mobiles Gerät übertragen und gespeichert werden soll, nicht zu groß werden zu lassen. Auf dem iPad können die konventionellen Röntgenbilder (Abb.9), Computertomografien, Kernspintomografien oder Sonografien (Abb. 10) dann in ausreichender Qualität sehr schnell netzunabhängig zur Anzeige gebracht werden. Die Qualität ist dabei zur kurzen Auffrischung des Gedächtnisses oder zur Demonstration, z. B. von Implantatlagen gegenüber dem Patienten, in der Regel voll ausreichend.

Neben der Möglichkeit, Befunde auf dem mobilen Gerät jederzeit verfügbar zu machen, lassen sich auch Funktionalitäten, wie in WhatsApp oder Facebook realisiert, auf die Medizin übertragen. So können Aufgaben erstellt und an eine definierte Person oder Personengruppe weitergeleitet werden. In der Anwendung Checkpad MED werden z.B. Aufgaben zum Patienten, die bei einer Visite entstehen, auswählbar gemacht. Um die Eingabe zu erleichtern, gibt es eine frei konfigurierbare Schnelltastatur, mit der die häufigsten Aufgaben nach Bereichen (als Beispiel hier Diagnostik, Therapie, Entlassungsplanung und Medizincontrolling) schnell dokumentiert werden können (Abb.11). Jeder Aufgabe kann dann ein Empfänger zugeordnet werden. Zusätzlich gibt es die Möglichkeit, die Priorität sowie ein Zieldatum festzulegen. Alle angelegten Aufgaben werden sofort bei Netzkontakt gegen die Server und die anderen mobilen Devices synchronisiert.

Neben dieser Funktionalität gibt es die Möglichkeit, einen Chat, ähnlich wie bei WhatsApp, zu beginnen. So kann z.B. mit einem erfahrenen Kollegen am Befund über die Beurteilung einzelner Parameter diskutiert werden. Diese Diskussion ist für alle an der Behandlung des Patienten beteiligten Kollegen sichtbar (Abb. 12).

Eine Funktionalität ähnlich der Facebook Timeline wurde ebenfalls realisiert. So gibt es die Möglichkeit, in einer konfigurierbaren Übersicht die wichtigsten Informationen, z.B. zur Vorstellung eines Patienten in einer Frühbesprechung, Röntgenkonferenz, Tumorkonferenz oder bei der Chefvisite, sehr schnell mit den wichtigsten Befunden, die im Schnellzugriff sind, zusammenzufassen (Abb. 13). Vorgeschlagene Bereiche sind dabei:

- Diagnosen

- Diagnostik

- Therapien

- Konsile

- Entlassungsplanung

- Aufgaben

- markierte Dokumente

- Notizen

- Laborwerte

Ein wichtiger Punkt bei mobilen Geräten im Krankenhaus ist natürlich der Datenschutz. Dabei sind verschiedene Angriffsszenarien zu bedenken. Ein Weg ist es, den Datenverkehr zwischen Server und Gerät zu belauschen. Dazu muss ein Zugang zum WLAN der Klinik erlangt werden. Das zweite Szenario ist das Auslesen der Daten aus dem Speicher des
Geräts, wenn Daten dort abgelegt sind. Solange nur Daten angezeigt werden, die auf anderen Servern gespeichert sind, ist nur der Verkehr sicher zu gestalten. Ein Verlust von Geräten ist z. B. bei einem Diebstahl zu beklagen. Da praktisch kein Krankenhaus über eine komplette flächendeckende WLAN-Ausleuchtung verfügt, gibt es aber immer Bereiche, in denen keine Daten angezeigt werden, wenn nur online gearbeitet werden kann.

Bei der Software Checkpad MED wurde daher primär die Entscheidung getroffen, dass alle Daten tatsächlich bei Netzwerkkontakt auf das Gerät übertragen werden. Der Vorteil ist, dass die Daten immer verfügbar sind und dass die Geschwindigkeit der Anzeige von Informationen extrem schnell ist. Ein weiterer Nachteil der reinen Online-Anwendungen ist auch, dass die Netzlast bei intensiver Nutzung sehr hoch sein kann, da z.B. jedes Röntgenbild für jede Ansicht einmal über das Netzwerk geschickt werden muss. Um dies zu vereinfachen, wird manchmal der Versuch des Cachings unternommen, wobei beachtet werden muss, dass die Daten nicht in einem unverschlüsselten Bereich auf dem Endgerät abgelegt werden, da dieser bei Geräteverlust relativ einfach ausgelesen werden könnte.

Bei Checkpad MED werden die Daten über eine SSL-Verbindung verschlüsselt an das Gerät übertragen und dort wiederum mehrfach verschlüsselt. Die erste Verschlüsselung nimmt Apple vor. Die zweite Verschlüsselung erfolgt mit der Nutzer-PIN des Geräts. Viele Kliniken verlangen dort einen 6-stelligen Code aus numerischen und alphanumerischen 


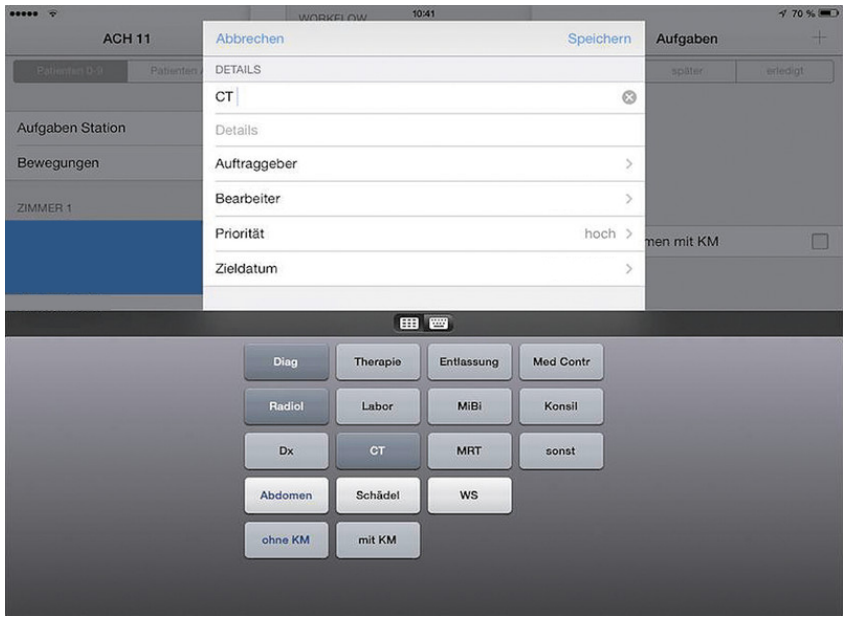

Abb. 11 Dokumentation von Aufgaben mittels Schnelltastatur auf dem iPad.

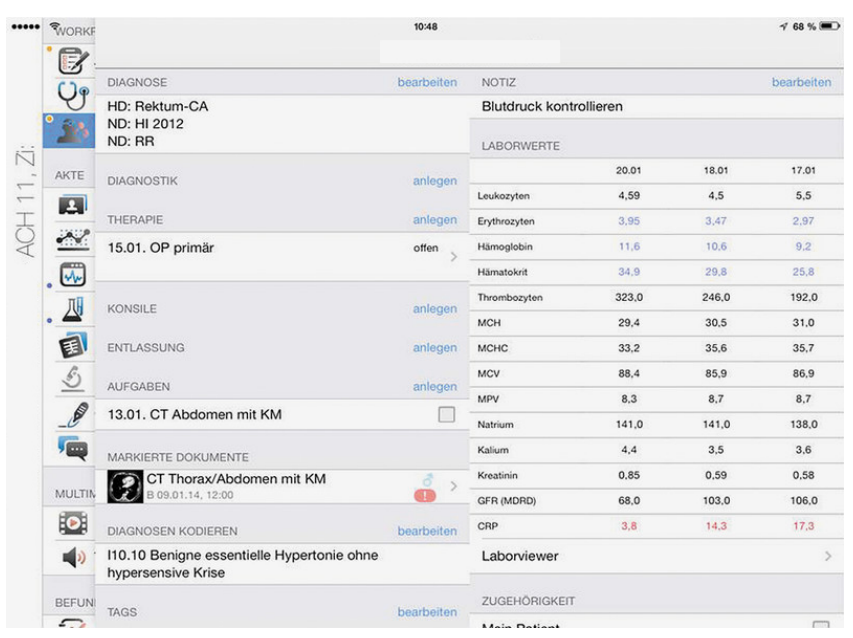

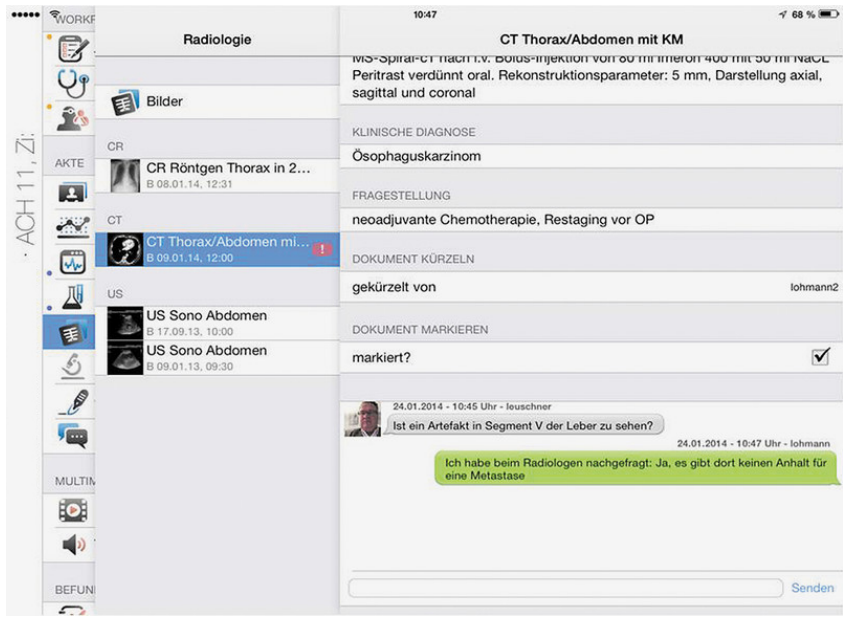

Abb. 12 Chat zu einem Befund auf dem iPad.

Abb. 13 Übersicht zum Patienten auf dem iPad.

Applikationsstart oder laufender Applikation gelöscht werden. Zusätzlich ist die Möglichkeit des sog. Geofencings eingebaut. Über Zuordnung, z.B. aller Hotspots in ein Koordinatensystem, weiß das System, wo sich jedes iPad befindet. Nun können Flächen definiert werden, in denen das Gerät arbeiten darf. Bei Verlassen dieser Flächen kann die Applikation automatisiert beendet werden und die Daten können automatisiert gelöscht werden. Zusätzlich besteht, z.B. über ein Mobile Device Management, die Möglichkeit der Fernlöschung der gesamten Geräte. Seit der Version 7 des iOS kann zudem eingestellt werden, dass Geräte danach auch in iTunes ohne die notwendigen Kennwörter nicht wieder aktiviert werden können. Damit sind gestohlene Geräte praktisch wertlos.

Neben der Verschlüsselung kommen weitere Technologien zum Einsatz. So ist einstellbar, nach welcher Zeit ohne Kontakt zum Server sämtliche Daten bei

\section{Diskussion}

Viele Arbeitsprozesse in der Medizin bedürfen der Zusammenarbeit verschiedener Beteiligter. Oft ist die Validierung einer Entscheidung durch eine erfahrenere Person notwendig. Dieser Prozess ist heute durch die synchrone Kommunikation per Telefon, durch gemeinsame Besprechungen oder Visiten oft recht mühsam und in der täglichen Arbeit nicht immer einfach unterzubringen. Die Folge ist, dass Entscheidungen nicht immer abgestimmt werden, was oft unnötige Kosten verursacht und in einigen Fällen auch zu negativen Folgen für den Patienten führen kann.

Die zuvor beschriebene Technologie kann z.B. für den gesamten Prozess der OP-Vorbereitung genutzt werden. Alle Beteiligten können dann jederzeit auf die zum Patienten schon vorhandenen Informationen zurückgreifen, um Entscheidungen zu treffen. Bestimmte Aufgaben können im Prozess auf der Zeitschiene verortet werden und so automatisiert zu einem vorab definierten Zeitpunkt in die Aufgabenliste einzelner Personen oder Funktionsgruppen geschrieben werden. Der Abstimmungsprozess zwischen verschiedenen Beteiligten kann so digital erfolgen und von den Personen z.B. in kurzen Pausen zwischen zwei anderen Tätigkeiten, wie zwei Operationen, stattfinden. Wir glauben, dass durch den Einsatz dieser modernen Technologie die Qualität der Patientenversorgung verbessert werden kann, da Entscheidungen so fast immer auf Grundlage von echten Informationen getroffen werden. Gleichzeitig wird sehr viel weniger vergessen, sodass auch Liegezeiten im Krankenhaus verkürzt wer- 
den können. Die Kommunikation zwischen den verschiedenen, an der Behandlung des Patienten beteiligten Personen wird vereinfacht und verbessert. Das führt dazu, dass weniger Aufgaben mehrfach erledigt werden und kann die tägliche Arbeitszeit, z.B. der Ärzte, signifikant verkürzen.

\section{Schlussfolgerung}

Mobile Devices können den Arbeitsalltag in den Kliniken signifikant verändern.

Gerade Prozesse wie OP-Vorbereitungen benötigen den Zugriff auf unterschiedlichste Informationsquellen. Bei den Entscheidungen, die in der Vorbereitung zu treffen sind, gibt es in der Regel viele Beteiligte. Neben der Informationsbereitstellung ist die Kommunikation mittels mobiler Geräte viel einfacher möglich. Damit halten Technologien, die im Alltag schon von sehr vielen Menschen genutzt werden (WhatsApp, iMessage oder Facebook), Einzug in den klinischen Alltag.

\section{Literatur}

${ }^{1}$ Gocke P, Debatin JF, Hrsg. IT im Krankenhaus Von der Theorie in die Umsetzung. Berlin: Medizinisch Wissenschaftliche Verlagsgesellschaft; 2011

${ }^{2}$ Herbig B, Büssing A, Hrsg. Informations- und Kommunikationstechnologien im Krankenhaus - Grundlagen, Umsetzung, Chancen und Risiken. Stuttgart: Schattauer; 2006
Dr. med. Rüdiger Lohmann

Geschäftsleitung

Lohmann \& Birkner Health Care

Consulting $\mathrm{GmbH}$

Alt-Reinickendorf 25

13407 Berlin

lohmann@lohmann-birkner.de

Prof. Dr. med. Almut Tempka

Leitende Ärztin Physiotherapie CVK, Oberärztin CMSC

Charité - Universitätsmedizin Berlin, Campus Virchow-Klinikum

Augustenburger Platz 1

13353 Berlin

Prof. Dr. med. Norbert Süikamp

Geschäftsführender Direktor

Department für Orthopädie und Traumatologie Universitätsklinikum Freiburg Hugstetter Straße 55 79106 Freiburg 